\title{
Multistate Resistive Switching Memory for Synaptic Memory Applications
}

Mrinal K. Hota ${ }^{1}$, Mohamed N. Hedhili ${ }^{1}$, Nimer Wehbe ${ }^{1}$, Martyn A. McLachlan ${ }^{2}$, and Husam N. Alshareef ${ }^{\text {* }}$

Dr. M. K. Hota, Dr. M. N. Hedhili, Dr. N. Wehbe, Prof. M. A. McLachlan, and Prof. H. N. Alshareef

${ }^{1}$ Physical Sciences and Engineering Division, King Abdullah University of Science \& Technology (KAUST), Thuwal 23955-6900, Saudi Arabia

${ }^{2}$ Department of Materials and Centre for Plastic Electronics, Imperial College London, London SW7 2AZ, United Kingdom

*E-mail: husam.alshareef@kaust.edu.sa

Keywords: memristors; mixed oxide; multi-bit memory; neuromorphic memory

\begin{abstract}
We report reproducible low bias bipolar resistive switching memory in $\mathrm{HfZnO}_{\mathrm{x}}$ based memristors. The modification of the concentration of oxygen vacancies in the ternary oxide film, which is facilitated by adding $\mathrm{ZnO}$ into $\mathrm{HfO}_{2}$, results in improved memory operation by the ternary oxide compared to the single binary oxides. A controlled multi-state memory operation was achieved by controlling current compliance and RESET stop voltages. A high DC cyclic stability up to 400 cycles in the multi-bit memory performance was observed. Conventional synaptic operation in terms of potentiation, depression plasticity and Ebbinghaus forgetting process were also studied. The memory mechanism is shown to originate from the migration of the Oxygen vacancies and the modulation of interfacial layers.
\end{abstract}

\section{Introduction}

Progress in digital electronic memory based on complementary metal-oxide-semiconductor (CMOS) technology has made a transformative leap in information storage during the last decade. 
However, adaptive intelligence learning has not yet been fully implemented in the binary system $(0$ and 1) based on classical von-Neumann computation paradigm, due to the limits of the hardware architecture ${ }^{[1]}$ In addition, Moore's law based CMOS scaling of semiconductor transistors is now facing significant barriers to achieving historical performance gains. ${ }^{[2-3]}$ Also, for 'real-time' complex computing (such as in 'artificial intelligence') the computation time increases exponentially with the input data size. As a result, it is extremely difficult (sometimes impossible) to perform such tasks with conventional computers. ${ }^{[4]}$ Hence, development of "next-generation" memory technology beyond the conventional digital logic, is an urgent technological requirement for handling the massive complex data that are generating every day in our society. In contrast, electronic synaptic memories have received tremendous interest in the scientific community and are considered to be essential building blocks in achieving artificial neural networks. ${ }^{[5]}$ The aim of these synaptic memories is to emulate the highly efficient real-time capabilities of biological information processes. ${ }^{[6]}$ Therefore, the challenge is to build brain-inspired, "neuromorphic" computational systems to mimic the biological brain for performing the energy-efficient and fault-tolerant computation. Moreover, it would combine memory and computation in a single electronics element. ${ }^{[7]}$

On the other hand, to increase the bit density and to reduce the cost per bit, memory devices with multilevel characteristics are required. ${ }^{[8]}$ Over the last decade, several attempts were made to demonstrate the multi-bit memory performance in different device architectures. Among these approaches, memristor (memory + resistor) based non-volatile memory devices are found to be promising for multi-level memory operations, due to their simple metal-insulator-metal capacitor structure, nanoscale scaling possibility, high speed and controlled multi-level conductance capability. ${ }^{[9]}$ Moreover, the analog-like gradual transition between different resistance levels under optimized conditions (input impulses) made it suitable for electronics synaptic memory applications to emulate the plasticity of biological synapses. ${ }^{[10]}$ It is reported that memristor devices based on different material systems adequately mimic different important neuromorphic properties, such as 
potentiation, depression, learning, forgetting, etc. ${ }^{[11-14]}$ Many novel materials including chalcogenides $\left(\mathrm{Ge}_{2} \mathrm{Sb}_{2} \mathrm{Te}_{5}\right), \mathrm{Ag} / \mathrm{Si}$ mixture, $\mathrm{AlO}_{\mathrm{x}} / \mathrm{HfO}_{\mathrm{x}}$ bi-layer, etc. are reported as memristive material to mimic the synaptic functions. ${ }^{[2,15-16]}$ Among different memristive materials, Hf-based oxide materials are found promising due to its excellent memory performance and compatibility with the present CMOS process technology. ${ }^{[17-19]}$ On the other hand, zinc oxide $(\mathrm{ZnO})$ is cost effective and multistate memory performance has already been reported in ZnO-based memristor devices. $^{[20]}$

In this study, we prepared an atomic layer deposition (ALD) deposited $\mathrm{HfZnO}_{\mathrm{x}}$ based memristor device for multistate and neuromorphic memory evaluation. To our knowledge, multistate and neuromorphic memory study in $\mathrm{HfZnO}_{\mathrm{x}}$ oxides have not yet been reported. The rational for choosing this composition is to combine the ability of $\mathrm{ZnO}$ to form oxygen vacancies (and related defects) relatively easily compared with $\mathrm{HfO}_{2}$, which is known to show resistive memory effect. The memory characteristics such as multistate current-voltage characteristics, multistate endurance, etc. at room temperature are discussed. We further exploit the multistate memristive properties of our memristor devices to demonstrate their synaptic functionalities. It was observed that the mixed oxide shows better multi-level memory performance as compare to individual oxide based memory devices.

\section{Results and discussion}

\subsection{Material Characterization}

The chemical compositions of the ALD deposited films were analyzed by X-ray photoelectron spectroscopy (XPS) analysis. The XPS spectra obtained from the as-deposited oxide films are shown in Figure 1. The binding energies were corrected considering the sample charging effect using the $\mathrm{C} 1 s$ line at $285 \mathrm{eV}$. All XPS spectra were deconvoluted using the standard Gaussian-Lorentzian function. However, before deconvolution of all spectra, Shirley background correction was applied to eliminate background signal. Figure 1a shows Hf $4 f$ core level spectra 
with two distinct peaks corresponding to the $\mathrm{Hf} 4 f_{7 / 2}$ and $\mathrm{Hf} 4 f_{5 / 2}$ at 16.7 and $18.4 \mathrm{eV}$, respectively. The spin-orbit splitting between $\mathrm{Hf} 4 f_{7 / 2}$ and $\mathrm{Hf} 4 f_{5 / 2}$ photoelectrons was found $1.7 \mathrm{eV}$. These two peaks represent the characteristics of $\mathrm{Hf}-\mathrm{O}$ bonding. ${ }^{[21]}$ In addition, no peaks at the lower energy side appeared in the spectra of the as-deposited films, suggesting that the Hf-Hf bonding state does not exist. ${ }^{[22]}$ The XPS spectra from the $\mathrm{Zn} 2 p$ core level is shown in Figure 1b. Two distinct peaks corresponding to $\mathrm{Zn} 2 p_{3 / 2}$ and $\mathrm{Zn} 2 p_{1 / 2}$ were found at 1022.2 and $1045.3 \mathrm{eV}$, respectively. It can be observed that the peak position for $\mathrm{Zn} 2 p_{3 / 2}$ and $\mathrm{Zn} 2 p_{1 / 2}$ were shifted towards the higher binding energy side by $0.7 \mathrm{eV}$, as compared to the $\mathrm{Zn} 2 p$ core level spectra obtained from the pure $\mathrm{ZnO}$ samples (Figure S1). On the other hand, the small peak at higher binding energy side in both $\mathrm{Zn}$ $2 p_{3 / 2}$ and $\mathrm{Zn} 2 p_{1 / 2}$ spectra may be due to the presence of zinc interstitial $\left(\mathrm{Zn}_{\mathrm{i}}\right)$ and/or $\mathrm{Zn}(\mathrm{OH})_{2}$ in the films. ${ }^{[23-24]}$ In order to investigate the effect of $\mathrm{ZnO}$ incorporation into $\mathrm{HfO}_{2}$ in term of oxygen vacancy $\left(V_{O}^{2+}\right)$ concentration, the $\mathrm{O} 1 s$ spectra obtained from both $\mathrm{HfO}_{2}$ and $\mathrm{HfZnO}_{\mathrm{x}}$ samples were studied. The asymmetric $\mathrm{O} 1 s$ spectra obtained from $\mathrm{HfO}_{2}$ and $\mathrm{HfZnO}_{\mathrm{x}}$ film are shown in Figure 1c and Figure 1d, respectively. It is observed that both spectra can be deconvoluted into three parts, which are defined as $\mathrm{O}_{\mathrm{I}}$ (at lower binding energy side), $\mathrm{O}_{\mathrm{II}}$ (at intermediate binding energy side) and $\mathrm{O}_{\text {III }}$ (at higher binding energy side). The peak belonging to $\mathrm{O}_{\mathrm{I}}$ (at $530.1 \mathrm{eV}$ for both $\mathrm{HfO}_{2}$ and $\mathrm{HfZnO}_{\mathrm{x}}$ ) is attributed to the $\mathrm{O}^{2-}$ ions, which are surrounded by either $\mathrm{Zn}$ of $\mathrm{Hf}$ atoms, i.e., $\mathrm{O}_{\mathrm{I}}$ spectrum can be assigned to the $\mathrm{Zn}-\mathrm{O}$ or $\mathrm{Hf}-\mathrm{O}$ bonds. ${ }^{[25]}$ The component at the intermediate binding energy, $\mathrm{O}_{\mathrm{II}}$ (at $530.7 \mathrm{eV}$ for both $\mathrm{HfO}_{2}$ and $\mathrm{HfZnO}_{\mathrm{x}}$ ) is associated with $\mathrm{O}^{2-}$ ions that are in oxygen-deficient regions within the oxide film, which signifies the existence of oxygen vacancies in the oxide films. ${ }^{[26]}$ From XPS compositional analysis, we find that the amount of oxygen vacancies $\left(V_{O}^{2+}\right)$ in $\mathrm{HfO}_{2}$ is $\sim 11.5 \%$, and it increases up to $\sim 21.1 \%$ after incorporation of $\mathrm{ZnO}$ into $\mathrm{HfO}_{2}$ matrix. The high binding energy component located at $\sim 532 \mathrm{eV}$ is usually attributed to the presence of loosely bound oxygen on the surface of the oxide film belonging to a specific species, e.g., $-\mathrm{CO}_{3}$ or adsorbed $\mathrm{H}_{2} \mathrm{O}$ or adsorbed $\mathrm{O}_{2} \cdot{ }^{\left[{ }^{[27]}\right.}$ However, the intensity of the $\mathrm{O}_{\text {I }}$ peak exceeds those of the $\mathrm{O}_{\mathrm{II}}$ and $\mathrm{O}_{\mathrm{III}}$ peaks, signifying the strong $\mathrm{Zn}-\mathrm{O}$, O-Hf-O, and $\mathrm{Zn}-\mathrm{O}-\mathrm{Hf}$ bonding in $\mathrm{HfZnO}_{\mathrm{x}}$ film. Hence, 
the XPS results reveal the formation of $\mathrm{HfZnO}_{\mathrm{x}}$ mixed oxide films and the increase in oxygen vacancy concentration after the incorporation of $\mathrm{Zn}$ into the $\mathrm{HfO}_{2}$ matrix.

Figure $2 \mathrm{a}$ and Figure $2 \mathrm{~b}$ show the dynamic secondary ion mass spectrometry (SIMS) depth profile of the $\mathrm{HfZnO}_{\mathrm{x}}$ based resistive switching memory device in its virgin and electroformed state, respectively, after removing the top Au electrodes. It is clearly observed that two 'bumps' are exhibited by the signal ascribed to TiO. These two 'bumps' indicate the presence of oxygen in the Ti overlayer, which may have diffused from the $\mathrm{HfZnO}_{x}$ layer. The intensity of the TiO signal, especially the first bump, is clearly enhanced when the sample was subjected to positive electroforming bias on the top electrode, i.e., at the electroformed state (the comparison is shown in Figure S2). The bump shape evidenced by the oxygen signal is consistent with the rise of the titanium oxide signal. In addition, the $\mathrm{ZnO}^{-}$and $\mathrm{HfO}^{2-}$ signals exhibit a slight, but visible, always decrease after applying a positive electroforming bias to the sample. The detection of more oxygen in the Titanium layer together with the slight decrease of the $\mathrm{HfO}^{2-}$ and $\mathrm{ZnO}^{-}$signals in the oxide layer is consistent with a diffusion of oxygen from the $\mathrm{HfZnO}_{\mathrm{x}}$ oxide layer to the Titanium layer as a consequence of the positive electroforming bias. The SIMS data confirm the existence of an interfacial $\mathrm{TiO}$ layer in between $\mathrm{Ti}$ and $\mathrm{HfZnO}_{\mathrm{x}}$ interface, which increases during the electroforming process. We believe that this interfacial layer has a direct impact on the resistive switching performance of the device.

\subsection{Resistive switching memory characterization}

Figure 3a shows DC current-voltage $(I-V)$ characteristics results measured by a typical DC double sweep mode at room temperature. It is found that the as-prepared sample requires an initial electrical forming by positive bias sweep up to $10 \mathrm{~V}$ with the current compliance (CC) at $70 \mu \mathrm{A}$ (right inset in Figure 3a). Initially, the device was highly insulating $\left(10^{8} \Omega\right.$ at $\left.0.5 \mathrm{~V}\right)$. However, during the electroforming, the resistance of the device transformed from $10^{8} \Omega$ to $10^{3} \Omega$ (hereafter the resistance is given at a read voltage of $+0.5 \mathrm{~V}$ ). The virgin state $I-V$ characteristics are shown in Figure $\mathbf{S 3}$ in the supplementary section. After the forming process, the $I-V$ data were obtained with 
the compliance current of $10 \mathrm{~mA}$ and $+2.5 /-2.5$ as stop voltage for either polarity side. It is observed that the SET, i.e., the transition from high resistance state (HRS) to low resistance state (LRS) and RESET, i.e., the transition from LRS to HRS process occurs at negative and positive polarity sides respectively, i.e., polarity-dependent resistive switching can be clearly seen. Once the SET or RESET operation performed, LRS or HRS were kept as long as the RESET or SET bias was applied again to the device. It can be observed that the order of the current level increases suddenly during the SET process, which signifies the formation of the conducting filaments. ${ }^{[28]}$ However, during the RESET process, the current decreases stepwise, which signifies the partial rupture of individual conducting filaments. Here, it can be noted that the memory devices with only $\mathrm{HfO}_{2}$ and $\mathrm{ZnO}$ as resistive switching layer did not show a good memory performance in our study, as presented in Figure S4a and Figure S4b. These results signify that there must be a role of oxygen vacancies that increases in the mixed oxide, $\mathrm{HfZnO}_{\mathrm{x}}$ after incorporating of $\mathrm{ZnO}$ in $\mathrm{HfO}_{2}$, as has been seen from the XPS results. Hence, we believe that mixed ternary oxides, such as $\mathrm{HfZnO}_{\mathrm{x}}$, are more suitable as compared to binary oxides for resistive switching memory application. The DC cyclic study of the memory performance is shown in Figure 3b. The resistance ratio between HRS and LRS is more than 10 times, which was maintained up to 400 cycles, and the distinguished binary states can be used to store the logic " 0 " or " 1 " respectively for memory application. Thus, these results signify the nonvolatile bipolar resistive switching memory effects in the present memristor device.

Next, we attempted to study the multistate memory possibility in $\mathrm{HfZnO}_{\mathrm{x}}$ based memristor devices. An adequate separation between LRS and HRS state may provide the possibility of multistate storage. It was observed that multistate memory characteristic can be achieved by controlling the current compliance (CC) during the SET processes (e.g. $10 \mathrm{~mA}$ and $30 \mathrm{~mA}$ ) and also by controlling the RESET stop voltage $\left(\mathrm{V}_{\text {stop }}\right)$ during the RESET process (e.g. 2, 2.5 and $\left.3 \mathrm{~V}\right)$. The multistate resistive switching memory characteristics obtained from $\mathrm{ITO} / \mathrm{HfZnO}_{\mathrm{x}} / \mathrm{Ti} / \mathrm{Au}$ memory structure are shown in Figure $3 \mathrm{c}$ and Figure $\mathbf{3 d}$ respectively by controlling $\mathrm{CC}$ and $\mathrm{V}_{\text {stop. }}$ It is 
observed that an apparent 3 level memory state obtained during CC control, whereas a 4 level memory state was found by controlling the RESET stop voltage. For convenience, we defined the memory states as “1", " 2 ", “3" and "4". It is also observed that controlling CC and $\mathrm{V}_{\text {stop }}$ could result in different resistances at the HRS whereas a common LRS states were shared. This type of memory performances are different compared to those published in the literature. It is reported that controlling CC, different LRS resistance level is possible, however here we obtained different HRS resistance levels. ${ }^{[29-30]}$ These results suggest that same type of conducting filaments (CFs) may form during the SET process with CC of 10 and $30 \mathrm{~mA}$. On the other hand, different resistance level at HRS implies that there may be few residual un-ruptured CFs within the memory film during the switching cycles. ${ }^{[30]}$ Therefore, by applying higher positive stop voltage or, the higher level of CC, these residual un-ruptured CFs will be ruptured, causing the device switching to a higher resistance state. It was observed that the memory states are very stable at each memory state. However, better control over multi-bit memory performance were achieved by controlling the $\mathrm{V}_{\text {stop }}$ as compared to controlling the CC.

To understand the conduction process during multi-level resistive switching in the ITO/HfZnO ${ }_{\mathrm{x}} / \mathrm{Ti} / \mathrm{Au}$ memory device, the charge transport mechanism should be carefully examined due to the complexity of the conduction process. The LRS current behavior in both negative and positive sides show linear dependency in the log-log scale (slope $\sim 1$ ) of the current-voltage characteristics, as shown in Figure S5a and Figure S5b. It signifies the existence of the Ohmic type conduction process which may be due to the presence of the conducting filaments that formed during the SET process. However, the current level in different HRS states show multiple conduction process in both CC and RESET stop voltage dependent methods, as shown inset of Figure 3c and Figure 3d. The current conduction at lower voltage regain (denoted as "A" and "C" in the inset of Figure 3c) in both HRS states ("2" and "3") during CC methods shows linear dependence in the log-log scale, which signifies the Ohmic conduction process. When the applied voltage is small, the number of injected electrons are smaller than that of the thermally generated 
free electrons, thus the current depends on the external bias and the conductivity of the material. Hence, the current follows, Ohmic conduction process. However, when voltage further increases current become non-linear with the applied voltage and the slope was found $\sim 1.4-1.5$ in regions "B" and "D". This type of conduction might be from the Poole-Frenkel (PF) conduction process. ${ }^{\text {[31- }}$ ${ }^{32]}$ The current conduction dominated by the PF conduction process strongly indicates the existence of a defective interfacial layer in between top electrode and the oxide layer. ${ }^{[33]}$ The existence of this defective interfacial layer, is also proved in the SIMS study (see Figure 2), as the existence of $\mathrm{TiO}_{\mathrm{x}}$ interlayer in between $\mathrm{Ti}$ and $\mathrm{HfZnO}_{\mathrm{x}}$. However, if the applied voltage further increases conducting filaments forms in between top and bottom electrodes and current increases abruptly and starts to follow filamentary conduction process. On the other hand, in the case of $\mathrm{V}_{\text {stop }}$ controlled process different HRS current level at low and intermediate voltage level shows Ohmic (region "I" and "IV") and PF (region "II" and "V") conduction process. The existence of the residue conducting filaments during the switching cycles is may be the origin of the Ohmic conduction process at low voltage region. The PF conduction in the higher voltage region signifies the existence of an interfacial layer at bottom electrode and the oxide layer. However, in the higher voltage region ("III" and "IV"), the slope in the current-voltage fitting become more then 2, which signifies trap controlled space charge limited conduction process (TC-SCLC). As applied voltage increases, the injected electrons also increases rapidly. As a result, few of the injected electrons might be trapped in different traps (mainly oxygen vacancies) present in the oxide film and others will contribute to the total current. Hence, this cyclic change of current transportation process leads to the different current level i.e., multiple memory states.

For a memory application, reliability is an important issue. To check the device stability in different memory states, the DC cyclic stability test was performed. Figure $3 \mathrm{e}$ shows different resistance level read at $500 \mathrm{mV}$ under $\mathrm{DC}$ cyclic condition by controlling $\mathrm{V}_{\text {stop. }}$. It is observed that stable resistive switching cycles among four-bit levels exist up to 400 cycles without any degradation. To obtain further evidence for the switching stability, a statistical analysis was made in 
the form of cumulative probability for all memory states, as shown in Figure 3f. It can be observed from Figure $3 \mathrm{f}$ that the memory states do not overlap with each other, which is mandatory for the multi-bit memory operation to avoid failure in data storage. These results highlight the feasibility of the $\mathrm{HfZnO}_{\mathrm{x}}$ as oxide resistive switching material for high density and multistate memory applications.

The multi-bit memory performance that was observed above can be utilized for synaptic memory operation. The resistive switching memory device and its memory mechanisms are very much similar to that of the biological synapse. A schematic drawing of a biological synapse is shown in Figure S6. An input voltage signal can result in a drift of oxygen vacancies inside the oxide film of a memristor device, which may form conducting filaments, resulting in conductivity change in the device. Similarly, the biological synapse changes its conductance as a result of firing of $\mathrm{Ca}^{2+}$ or $\mathrm{Na}^{2+}$ ions from a 'pre-neuron' as a result of impulses signal. These ions diffuse through the nanogap junction, i.e., the synapse and received by the dendrites of a 'post-neuron'. To emulate the functions of a biological synapse, an analog memory with multiple memory states in between HRS and LRS is necessary. Figure $4 \mathrm{a}$ and Figure $4 \mathrm{~b}$ shows that the device conductance (current) continuously increases or decreases due to the successive DC negative or positive voltage double sweep operations, respectively. -3 and +3 were applied as the stop voltage during the negative and positive voltage sweep respectively with no current compliance was enforced on the samples. Here, the increase (SET) and decrease (RESET) of the device conductance is similar to the potentiation and depression, respectively, in biological synapses. ${ }^{[34]}$ These devices can be triggered to a high conductivity state by using consecutive moderate inputs and also could be depressed from the high conductive state after the application of appropriate consecutive strong inputs. It is speculated that formation of multiple filaments or increasing in filament diameter/number during consecutive DC negative sweeping leads to the gradual increase in current in the SET process. ${ }^{[35]}$ On the other hand, a partial rupture of multiple filaments and shrinking in the filament diameter in the middle due to the Joule heating effect may cause in gradual RESET process. ${ }^{[36]}$ Here, it may be noted that 
continuously increasing or decreasing current between the intermediate memory states without going back to the original memory state is crucial for the electronic synapse application to mimic the biological synapse.

This gradual conductance modification behavior of our electronics synapse devices was also studied in pulse stimulus mode, as shown in Figure 4c, which represents the synaptic weight modification in response to a potentiating or depressing stimulus. During the application of pulse programming, no compliance current was applied, which may eliminate the use of a current limiter in this memory device. For a stable synaptic weight modification, a proper pulse scheme is required. Here a $-1.5 \mathrm{~V}, 50 \mathrm{~ms}$ wide pulse was applied to the memory device repeatedly for 15 times and the current was measured after each excitation at a read voltage of $+500 \mathrm{mV}$. It can be observed that a gradual SET process is performed. After that, a $+1.5 \mathrm{~V} 50 \mathrm{~ms}$ wide pulse was repeatedly applied, and a continuous RESET process achieved. It is assumed that defects, mainly oxygen vacancies in the oxide material would increase due to the application of continuous SET pulses, which would lead to a lower device resistance. On the other hand, continuous increase of RESET pulse amplitude would recover defects resulting in a lower value of current. Hence, the change of defect concentration may lead to the synaptic weight modification. To understand this, it is necessary to study the transient current behavior as a function of the applied bias. It should be mentioned that during a typical voltage sweep, each bias point is applied to the device for a finite time before a measurement is done. This short time stress during each bias point leads to increase or decrease the conductivity of the device. ${ }^{[37]}$ In this study, constant voltage stressing (CVS) was applied with increasing amplitudes of bias and corresponding stress-induced leakage current (SILC) was recorded. Negative CVS with increasing voltages amplitude ranging from $-0.5 \mathrm{~V}$ to $-1.5 \mathrm{~V}$ and positive CVS with increasing voltages amplitude from +0.5 to $+1.5 \mathrm{~V}$ was applied to the device, as shown in Figure 4d and Figure 4e, respectively. It can be seen that no significant change in the current was observed when the device was stressed at $-0.5 \mathrm{~V} /+0.5 \mathrm{~V}$. This indicates that the activation of SILC and hence memory performance requires a minimum electric field. 
For an electronics synaptic device, it is important to emulate the Ebbinghaus forgetting process. It is the process by which memorization occurs on a finite time scale, and the memorization varies from volatile to permanent memory state. ${ }^{[38]}$ The current fading process was systemically investigated after a sequence of input pulses to mimic the biological forgetting process. Figure $4 \mathrm{f}$ shows different current decay scheme after the application of different strength of input stimulus. An instantaneous increase in the current amplitude can be observed immediately after each input pulse. This instantaneous increase in current level, i.e., the conductance of the device is analogous to the memorization process of the human brain during the short term memory events through a stimulation/impression. ${ }^{[13]}$ The conductivity of the device, i.e., the current start decaying gradually after each instantaneous increase in current level, i.e., in between two consecutive pulses. This decaying process is analogous to the memory loss in human memory immediately after input stimulation. However, it is also observed that the overall conductivity of the device increases gradually with the application of the potentiation pulses, and conductivity of the device decreases gradually during the application of the depression pulses. This phenomenon is similar to that of memory enhancement or detraction in the human brain through repeated simulations. These changes in the conductivity state of the device suggest a multi-store memory behavior of human memory. ${ }^{[38-39]}$ This current behavior may be due to the forming and deforming of the local conducting filaments. This study also signifies that multiple resistance states can be obtained by applying voltage pulses with different amplitudes. The continuous increase in current level during the application of the successive negative pulses, while continuous decreases in current level due to the application of the successive positive pulses analogous to the potentiation and depression phenomenon in a biological synapse.

The mechanism of the multi-state memory performance in $\mathrm{ITO} / \mathrm{HfZnO}_{\mathrm{x}} / \mathrm{Ti} / \mathrm{Au}$ memory structure can be explained by considering the conductive filament model and interface modulation. A schematic illustration of the multi-state memory mechanism in different conditions is shown in Figure 5. In the present case, the electronegativity of $\mathrm{Zn}$ is 1.65 which is higher than $\mathrm{Hf}$ (1.3). As a 
result, it is expected that a drift of $\mathrm{O}^{2-}$ ions towards the $\mathrm{Zn}$ side will take place, resulting in the creation of more oxygen vacancies $\left(V_{O}^{2+}\right)$ in the $\mathrm{HfZnO}_{\mathrm{x}}$ oxide film, as compared to pure $\mathrm{HfO}_{2}$ (as evidence from the XPS results shown Figure 1). In addition, after the deposition of Ti electrode, $O^{2-}$ from the oxide layer, will also drift towards Ti (electronegativity of 1.54) electrode and form $\mathrm{TiO}_{\mathrm{x}}$ interfacial layer by an oxidation process, which results in further increment of oxygen vacancies in the oxide layer. The thickness of this interfacial layer $\left(\mathrm{TiO}_{\mathrm{x}}\right)$ increases during the electroforming process (as evidence from the SIMS results shown in Figure 2). In the present case, a high positive bias was applied as the electroforming voltage, which causes to drift a large number of $\mathrm{O}^{2-}$ towards the $\mathrm{Ti}$ electrode resulting in increasing of $\mathrm{TiO}_{\mathrm{x}}$ interfacial layer. This types of the interfacial layer are normally oxygen deficient and can serve as an additional resistive switching layer. $^{[40]}$ However, this interfacial layer may serve as series resistance and hinders the electron injection into the oxide layer as soon as it forms. ${ }^{[41]}$ As a result, RESET process was observed at positive bias side. After the forming process, when the negative bias was applied to the top electrode, the oxygen vacancies $\left(V_{O}^{2+}\right)$ in both $\mathrm{TiO}_{\mathrm{x}}$ and $\mathrm{HfZnO}_{\mathrm{x}}$ oxide layers (initially, $V_{O}^{2+}$ are randomly distributed in the oxide films, as shown Figure 5a) start to drift towards the top electrode and at SET bias conducting filaments (a bridge of $V_{O}^{2+}$ ) forms from the bottom electrode to the top electrode, as shown in Figure 5b. In this condition, adjusting the current compliance, the formation of the numbers of conducting filaments or the drift motion of electrons, i.e., current level can be controlled. Hence, different current levels, i.e., different memory states are possible. Under the application of the negative bias on the top electrode, not only $V_{O}^{2+}$, but at the same time a drift motion of active $\mathrm{Sn}^{4+}$ ions from ITO bottom electrode to the oxide layer is also possible. These $\mathrm{Sn}^{4+}$ ions may partially react with $\mathrm{O}^{2-}$ in the oxide layer, near to the bottom electrode, would form the $\mathrm{Sn}^{2+}$ ions $\left(\mathrm{Sn}^{4+}+\mathrm{O}^{2-} \rightarrow \mathrm{Sn}^{2+}+\mathrm{O}_{0}\right.$, where $\mathrm{O}_{0}$ is the neutral oxygen molecules), which may form an interfacial layer (as also confirmed from the conduction mechanism mentioned above) near the bottom electrode along with rest of the $O^{2-}$ ions. ${ }^{[32]}$ These interfacial layer offers an internal 
resistance which helps in the controlling of the CC process, as this types of internal resistance layer leads to self-complince effect in the resistive swithcing memory devices. ${ }^{[42]}$ The reaction process of $\mathrm{Sn}^{4+}$ with $\mathrm{O}^{2-}$ enhances the $\mathrm{O}^{2-}$ absorption process into ITO, resulting in the enhancement of $V_{O}^{2+}$ generation process in oxide film. On the other hand, by adjusting the RESET voltage, the rupture process of the conducting filaments can also be controlled. The oxygen vacancies from the $\mathrm{TiO}_{\mathrm{x}}$ and $\mathrm{HfZnO}_{\mathrm{x}}$ layers start to deplete gradually as the RESET bias (positive) increases, resulting in gradual rupture of the conducting filaments. During the rupture process of the filaments, Joule heating effect also plays an important role. As RESET stop bias increases, the rupture process as a result of increasing the Joule heating effect increases. In the intermediate memory state, few conducting filaments may rupture, or a partial rupture of the conducting filaments may be possible as shown in Figure 5c. In addition, under the application of the positive bias on the top electrode, the internal resistance layer (accumulation of $\mathrm{Sn}^{2+}$ and $\mathrm{O}^{2-}$ ions) also annihilate gradualy with the increasing of the positive bias on the top electrode. Hence, the memory device start to follow RESET stop voltage. As a result, different intermediate HRS states achieved based on different $\mathrm{V}_{\text {stop }}$. After application of sufficient RESET voltage, when the conducting filaments rupture such a way that the bridge between the top and bottom electrode annihilated, the device come back to its fully HRS state, as shown in Figure 5d. Hence, it can be concluded that memristor devices with $\mathrm{HfZnO}_{\mathrm{x}}$ active layer are potentially useful materials for future multi-state and synaptic memory applications.

\section{Conclusion}

In conclusion, we successfully demonstrated multistate memory operation with $\mathrm{HfZnO}_{\mathrm{x}}$ as a memristive layer for neuromorphic memory applications. A controlled 4-bit memory operation was obtained by controlling the RESET voltage, which holds up to 400 sweeping cycles. On the basis of the understanding of the multi-bit memory operation, we have mimicked few conventional synaptic activities, such as potentiation, depression plasticity and Ebbinghaus forgetting process. The modification of the concentration of oxygen vacancies in the ternary oxide film, which is facilitated 
by adding $\mathrm{ZnO}$ into $\mathrm{HfO}_{2}$, results in promising memory operation. These findings open up opportunities to explore such ternary material systems not only for the multi-bit non-volatile memory operation but also for the next generation synaptic memory applications.

\section{Experimental Section}

The memristor was composed of ALD deposited $\mathrm{HfZnO}_{\mathrm{x}}$ mixed ternary oxide as a memristive layer sandwiched between ITO as bottom electrode layer and Ti/Au as the top electrode layer. Before the oxide deposition, the ITO $(\sim 100 \mathrm{~nm})$ coated glass substrates (commercially purchased) were sequentially cleaned with acetone, 2-propanol and DI water in an ultrasonic bath for about 5 min each. The mixed $\mathrm{HfZnO}_{\mathrm{x}}$ layer with $\sim 30 \% \mathrm{Zn}$ doping was deposited by ALD (Cambridge Nanotech) at $160{ }^{\circ} \mathrm{C}$ (substrate temperature) with Tetrakis (dimethylamido) hafnium (IV) and diethylzinc precursors with deionized water as an oxygen source. During ALD deposition process, hafnium precursor was heated to $75{ }^{\circ} \mathrm{C}$, and temperature of the Zinc precursor was maintained at room temperature. Total 400 ALD cycles were used for the deposition of the oxide layer, where every ten deposition cycles consist of $7 \mathrm{HfO}_{2}$ cycles followed by $3 \mathrm{ZnO}$ deposition cycles. Separate $\mathrm{HfO}_{2}$ and $\mathrm{ZnO}$ samples was also prepared by ALD to check the change in chemical composition of $\mathrm{HfO}_{2}$ after the incorporation of $\mathrm{ZnO}$. Finally, a bilayer of $\mathrm{Ti}(\sim 30 \mathrm{~nm}) / \mathrm{Au}(\sim 70 \mathrm{~nm})$ was deposited by e-beam evaporation through a shadow mask to pattern circular top electrodes of area $7.85 \times 10^{-5}$ $\mathrm{cm}^{2}$ each. Two separate devices replacing $\mathrm{HfZnO}_{\mathrm{x}}$ with $\mathrm{HfO}_{2}$ and $\mathrm{ZnO}$ of same thickness was prepared to compare the memory performance. The chemical composition and the structural analysis of the oxide films were performed using X-ray photoelectron spectroscopy (XPS) in a Kratos Axis Ultra DLD spectrometer equipped with a monochromatic Al $\mathrm{K}_{\alpha}$ X-ray source $(\mathrm{h} v=1486.6 \mathrm{eV})$ operating at $150 \mathrm{~W}$ with a multichannel plate and delay line detector under a vacuum of $10^{-10}$ Torr. The depth profiling experiments were acquired on a SIMS instrument from Hiden Analytical company (Warrington-UK) operated under ultra-high vacuum conditions $\left(10^{-9}\right.$ Torr). An argon beam of $2 \mathrm{KeV}$ was used to conduct the measurement. In order to avoid the edge 
effect, the SIMS data were collected from a small area of $50 \times 50 \mu \mathrm{m}^{2}$ centered in the middle of the eroded region. The electrical properties were measured using Keithley 4200 Semiconductor parameter analyzer in combination with a semi-automatic probe station from Cascade Microtech in voltage sweeping and pulse measurement mode at room temperature. All of the operating voltages or pulses were applied to the top electrode (Ti/Au) while ITO, the bottom electrode was grounded.

\section{Supporting Information}

Supporting Information is available from the Wiley Online Library or from the author.

\section{Acknowledgements}

Research reported in this publication was supported by King Abdullah University of Science and Technology (KAUST). The authors also thank Dr. Pradipta Nayak, the core laboratory staff, and the imaging and characterization staff at KAUST.

Received: ((will be filled in by the editorial staff))

Revised: ((will be filled in by the editorial staff)) Published online: ((will be filled in by the editorial staff)) 


\section{Reference}

[1] P. A. Merolla, J. V. Arthur, R. Alvarez-Icaza, A. S. Cassidy, J. Sawada, F. Akopyan, B. L. Jackson, N. Imam, C. Guo, Y. Nakamura, B. Brezzo, I. Vo, S. K. Esser, R. Appuswamy, B. Taba, A. Amir, M. D. Flickner, W. P. Risk, R. Manohar, D. S. Modha, Science 2014, 345, 668.

[2] D. Kuzum, R. G. D. Jeyasingh, B. Lee, H.-S. P. Wong, Nano Lett. 2012, 12, 2179.

[3] E. M. Vogel, Nat. Nanotechnol. 2007, 2, 25.

[4] J. M. Borwein, P. B. Borwein, Pi and the AGM: a study in analytic number theory and computational complexity; Wiley: New York, 1998.

[5] D. B. Strukov, Nature 2011, 476, 403.

[6] J. H. B. Wijekoon, P. Dudek, Neural Networks 2008, 21, 524.

[7] Y. Matveyev, K. Egorov, A. Markeev, A. Zenkevich, J. Appl. Phys. 2015, 117, 044901.

[8] D. Kuzum, S. Yu, H.-S. P. Wong, Nanotechnology 2013, 24, 382001.

[9] D. B. Strukov, G. S. Snider, D. R. Stewart, R. S. Williams, Nature 2008, 453, 80.

[10] S. Song, K. D. Miller, L. F. Abbott, Nature Neurosci. 2000, 3, 919.

[11] S. Yu, B. Gao, Z. Fang, H. Yu, J. Kang, H.-S. P. Wong, Adv. Mater. 2013, 25, 1774.

[12] Y. Li, Y. Zhong, J. Zhang, L. Xu, Q. Wang, H. Sun, H. Tong, X. Cheng, X. Miao, Sci. Rep. 2014, 4, 4906.

[13] S. G. Hu, Y. Liu, T. P. Chen, Z. Liu, Q. Yu, L. J. Deng, Y. Yin, S. Hosaka, Appl. Phys. Lett. 2013, 102, 183510.

[14] F. Alibart, E. Zamanidoost, D. B. Strukov, Nat. Commun. 2013, 4, 2072.

[15] S. H. Jo, T. Chang, I. Ebong, B. B. Bhadviya, P. Mazumder, W. Lu, Nano. Lett. 2010, 10, 1297.

[16] S. Yu, Yi Wu, R. Jeyasingh, D. Kuzum, H.-S. P. Wong, IEEE Trans. Electron Dev. 2011, 58, 2729.

[17] M. K. Hota, C. Mukherjee, T. Das, C. K. Maiti, ECS J. Solid State Sci. Technol. 2012, 1, N149. 
[18] H. Y. Lee, Y. S. Chen, P. S. Chen, P. Y. Gu, Y. Y. Hsu, S. M. Wang, W. H. Liu, C. H. Tsai, S. S. Sheu, P. C. Chiang, W. P. Lin, C. H. Lin, W. S. Chen, F. T. Chen, C. H. Lien, M.-J. Tsai, in IEDM Tech. Dig. 2010, 460.

[19] M. K. Hota, C. K. Sarkar, C. K. Maiti, Semicond. Sci. Technol. 2012, 27, 085002.

[20] Y. Han, K. Cho, S. Kim, Microelectron. Eng. 2011, 88, 2608.

[21] M. K. Hota, S. Mallik, C. K. Sarkar, S. Varma, C. K. Maiti, Jpn. J. Appl. Phys. 2011, 50, 101101

[22] Y. Geng, Z.-Y. Xie, W. Yang, S.-S. Xu, Q.-Q. Sun, S.-J. Ding, H.-L. Lu, D. W. Zhang, Surf. Coatings Technol. 2013, 232, 41.

[23] E. Guziewicz, M. Godlewski, L. Wachnicki, T. A. Krajewski, G. Luka, S. Gieraltowska, R. Jakiela, A. Stonert, W. Lisowski, M. Krawczyk, J. W. Sobczak, A. Jablonski, Semicond. Sci. Technol. 2012, 27, 074011.

[24] M. Wang, L.Jiang, E. J. Kim, S. H. Hahn, RSC Adv. 2015, 5, 87496.

[25] M. Chen, X. Wang, Y. H. Yu, Z. L. Pei, X. D. Bai, C. Sun, R. F. Huang, L. S. Wen, Appl. Surf. Sci. 2000, 158, 134.

[26] P.-T. Hsieh, Y.-C. Chen, K.-S. Kao, C.-M. Wang, Appl. Phys. A 2008, 90, 317.

[27] M. Chen, Z. L. Pei, C. Sun, L.S. Wen, X. Wang, J. Cryst. Growth 2000, 220, 254.

[28] M. K. Hota, M. K. Bera, S. Verma, C. K. Maiti, Thin Solid Films 2012, 520, 6648.

[29] D. L. Xu, Y. Xiong, M. H. Tang, B. W. Zeng, Y. G. Xiao, Appl. Phys. Lett. 2014, 104, 183501.

[30] S. Y. Wang, C. W. Huang, D. Y. Lee, T. Y. Tseng, T. C. Chang, J. Appl. Phys. 2010, 108, 114110.

[31] S. Jung, J. Kong, S. Song, K. Lee, T. Lee, H. Hwang, S. Jeond, J. Electrochem. Soc. 2010, 157, H1042.

[32] M. K. Hota, D. H. Nagaraju, M. N. Hedhili, H. N. Alshareef, Appl. Phys. Lett. 2015, 107, 163106.

[33] S.-L. Li, D. S. Shang, J. Li, J. L. Gang, D. N. Zheng, J. Appl. Phys. 2009, 105, 033710. 
[34] S. Yu, Y. Wu, R. Jeyasingh, D. Kuzum, H.-S. P. Wong, IEEE Trans. Electron Dev. 2011, 58, 2729.

[35] L. Goux, Y.-Y. Chen, L. Pantisano, X.-P. Wang, G. Groeseneken, M. Jurczak, D. J. Wouters, Electrochem. Solid-State Lett. 2010, 13, G54.

[36] M. J. Rozenberg, M. J. Sánchez, R. Weht, C. Acha, F. Gomez-Marlasca, P. Levy, Phys. Rev. B 2010, $81,115101$.

[37] S. Mandal, A. El-Amin, K. Alexander, B. Rajendran, R. Jha, Sci. Rep. 2014, 4, 5333.

[38] R. Yang, K. Terabe, G. Liu, T. Tsuruoka, T. Hasegawa, J. K. Gimzewski, M. Aono, ACS nano, 2012. 6,9515 .

[39] R. C. Atkinson, R. M. Shiffrin, In The Psychology of Learning and Motivation: Advances in Research and Theory (Eds: K. W. Spence, J. T. Spence) Academic: New York, 1968.

[40] H. Jeon, J. Park, H. Kim, H. Kim, W. Jang, H. Song, H. Jeon, J. Vac. Sci. Technol. B 2015, 33, 051204.

[41] Y. Lai, P. Xin, S. Cheng, J. Yu, Q. Zheng, Appl. Phys. Lett. 2015, 106, 031603.

[42] W. Bai, R. Huang, Y. Cai, Y. Tang, X. Zhang, Y. Wang, IEEE Electron Device Lett. 2013, 34, 223. 


\section{Figure captions:}

Figure 1: X-ray photoelectron spectroscopy spectra obtained from (a) $\mathrm{Hf} 4 f$, (b) $\mathrm{Zn} 2 p$ core level. The O1s spectra obtained from (c) $\mathrm{HfO}_{2}$ and (d) $\mathrm{HfZnO}_{\mathrm{x}}$ samples. It is found that the amount of oxygen vacancies increases after incorporation of $\mathrm{Zn}$ into $\mathrm{HfO}_{2}$ matrix.

Figure 2: SIMS results for the device in its (a) virgin state and (b) after electroforming process.

Figure 3: (a) Typical bipolar memristive $I-V$ characteristics measured from $\mathrm{ITO} / \mathrm{HfZnO} \mathrm{x} / \mathrm{Ti} / \mathrm{Au}$ memory structure. The forming process by controlling the current compliance is shown inset. (b) Switching endurance characteristics obtained from the same device up to 400 cycles. Multi-state memory characteristics obtained from $\mathrm{ITO} / \mathrm{HfZnO}_{\mathrm{x}} / \mathrm{Ti} / \mathrm{Au}$ memory structure by controlling (c) current compliance and (d) RESET voltage. The current conduction mechanism described in the negative (inset of (c)) and positive (inset of (d)) sides respectively. (e) Multi-state DC cyclic stability test measured by controlling RESET voltage. Stable 4-bit memory was obtained up to 400 cycles, and (f) cumulative probabilities of resistance associated with the four resistive states.

Figure 4: $\mathrm{I}-\mathrm{V}$ characteristics of the $\mathrm{ITO} / \mathrm{HfZnO}_{\mathrm{x}} / \mathrm{Ti} / \mathrm{Au}$ memristor device at (a) negative and (b) positive continuous sweeping bias. The voltage sweep range is from 0 to -3 or $3 \mathrm{~V}$ then back to 0 , and the time for a sweep cycle is $\sim 2 \mathrm{~s}$. The device conductivity continuously increases or decreases during the negative or positive voltage sweeps, respectively. (c) Potentiating $(-1.5 \mathrm{~V})$ pulses of 50 ms width were applied for first 15 cycles. Then depressing $(+1.5 \mathrm{~V})$ pulses $50 \mathrm{~ms}$ width were applied for next 15 cycles. Constant voltage stressing with different voltage amplitudes for (d) negative and (e) positive bias. (f) Retention characteristics studied by applying positive and negative voltage pulses of increasing amplitude. 
Figure 5: Schematic of memory mechanism in $\mathrm{HfZnO}_{\mathrm{x}}$ based memristors in its (a) initial condition, (b) SET condition, (c) intermediate memory condition, and (d) fully HRS condition. 
ToC

Multistate Memristors for Neuromorphic Memory Applications

Mrinal K. Hota, Mohamed N. Hedhili, Nimer Wehbe, Martyn A. McLachlan, and Husam N. Alshareef

ALD deposited mixed oxide, $\mathrm{HfZnO}_{\mathrm{x}}$ was used as resistive swithcing media to demonstrate the multi-bit memory performance and the synaptic memory effects in ITO/HfZnO $/ \mathrm{Ti} / \mathrm{Au}$ memristor devices. A stable memory performance was acheiveed due to the enhancement of the oxygen vacancies in the oxide film.

Table of Contents figure

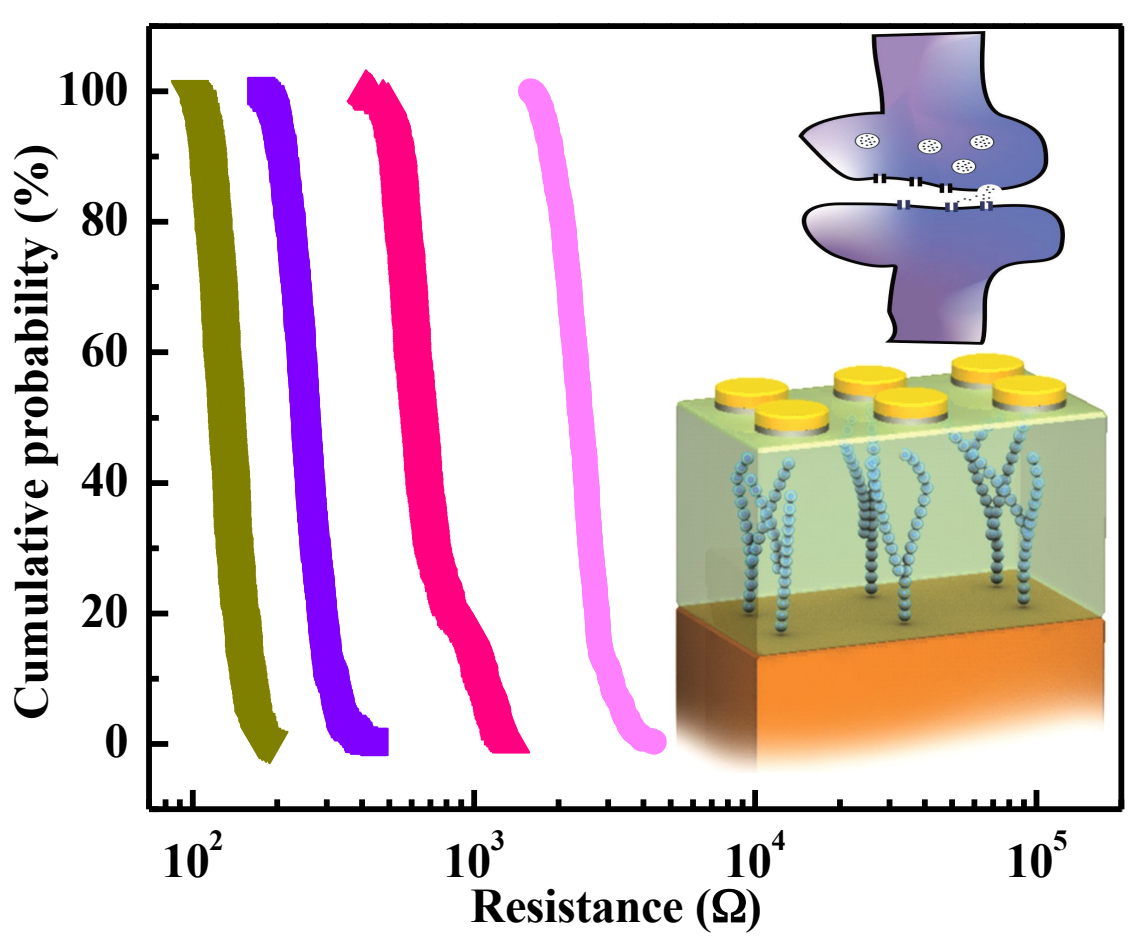


Figure 1.
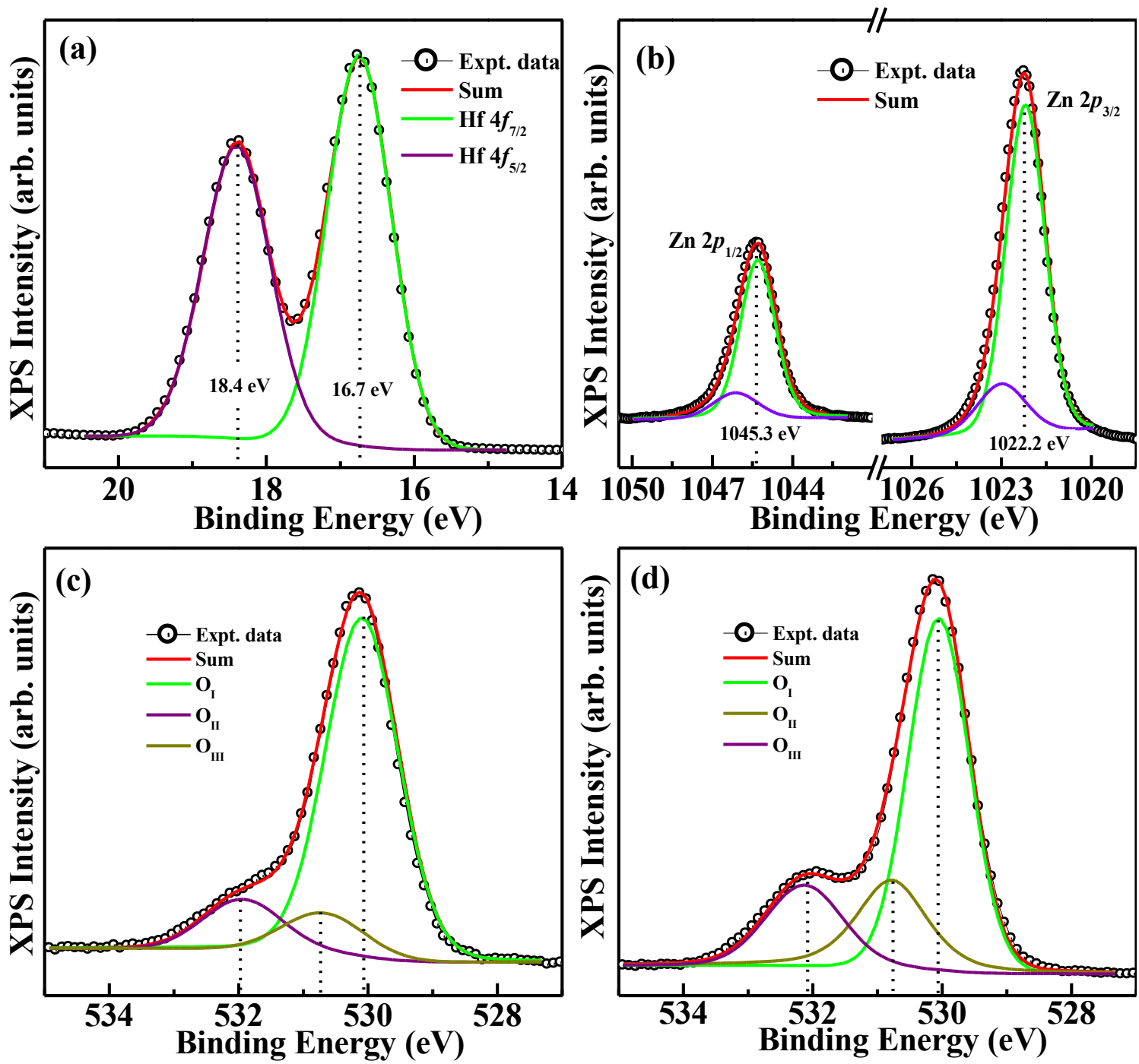
Figure 2.
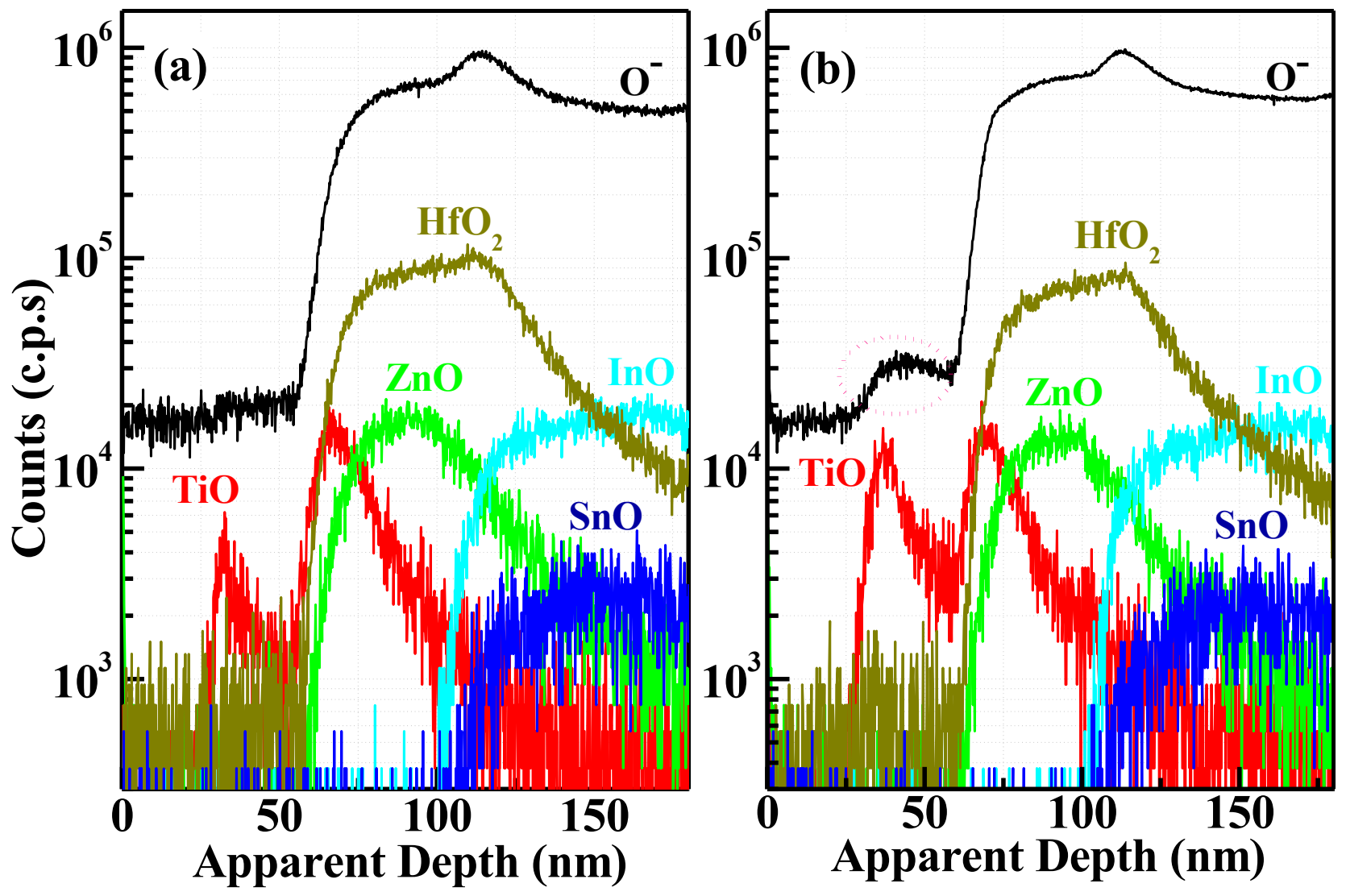
Figure 3.
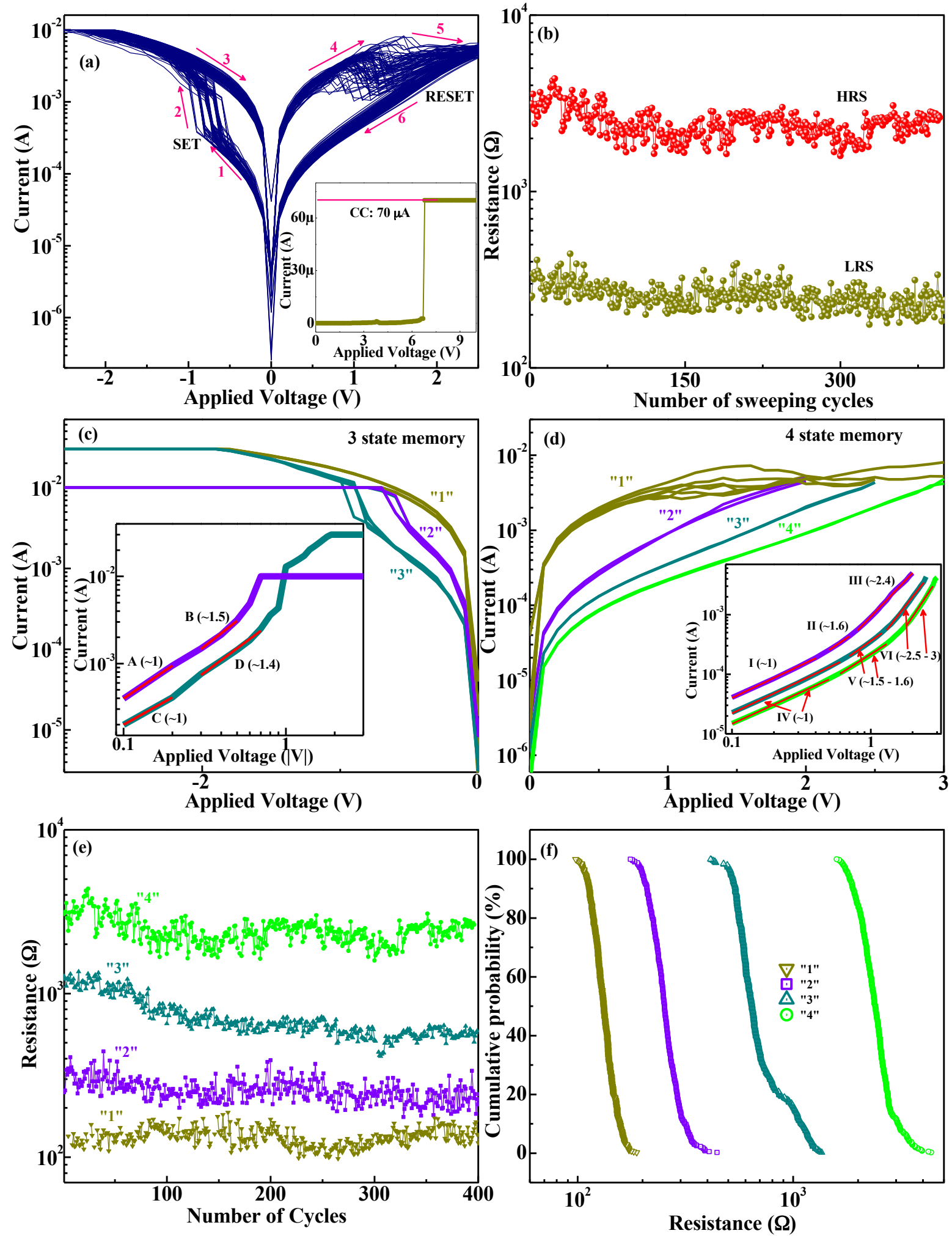
Figure 4.
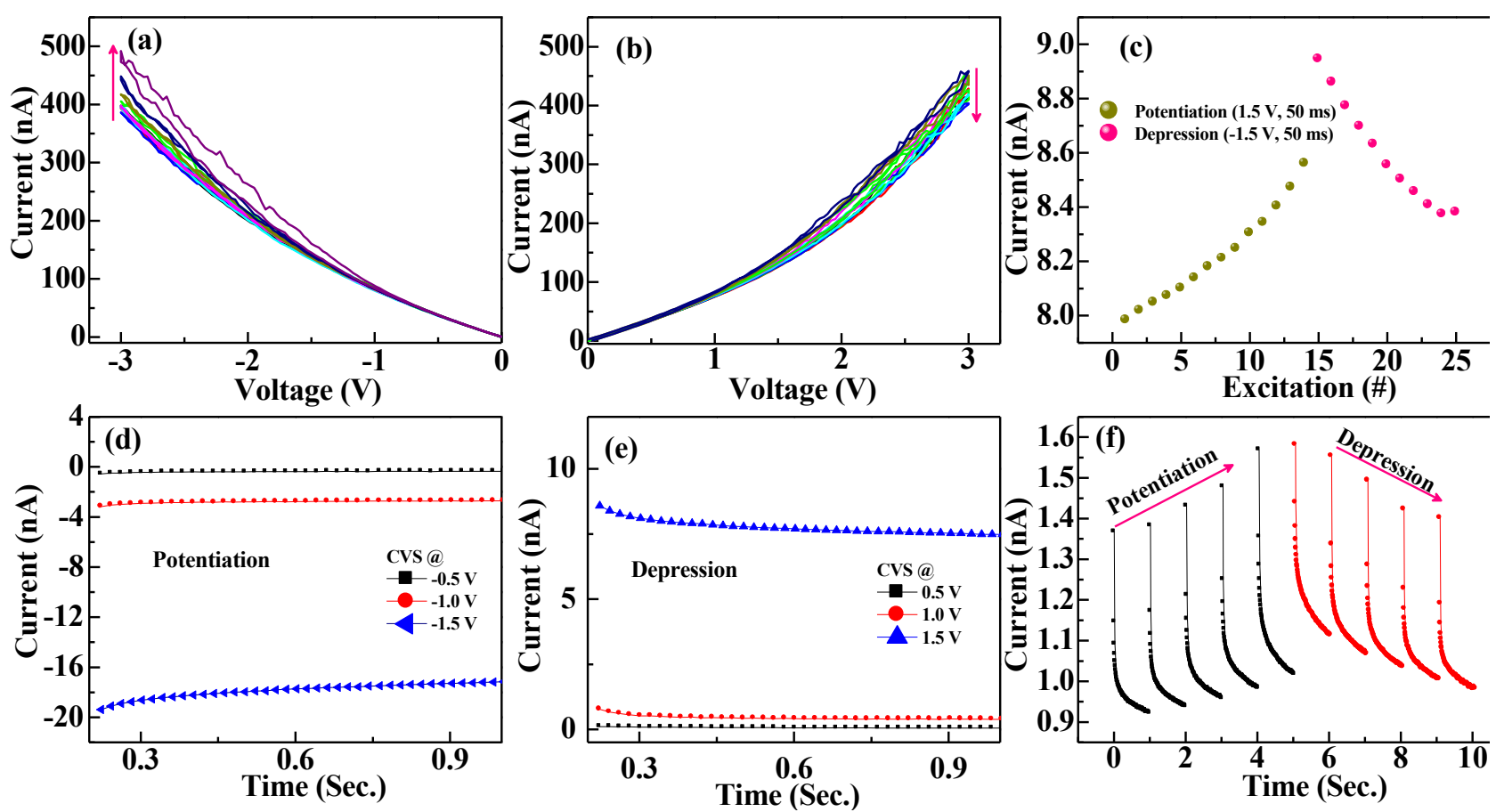
Figure 4

- Oxygen vacancy

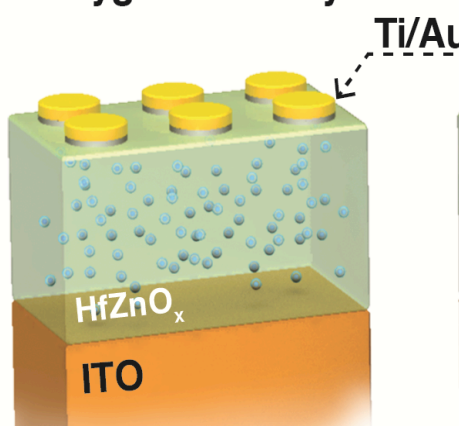

(a)

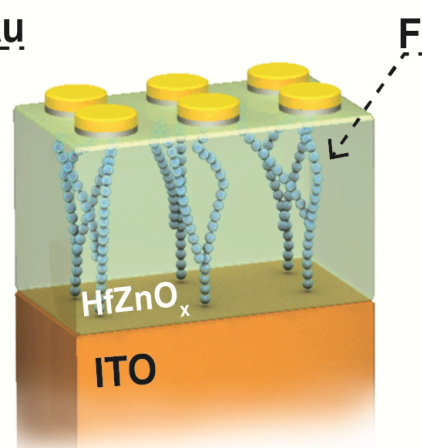

(b)
Filamenent

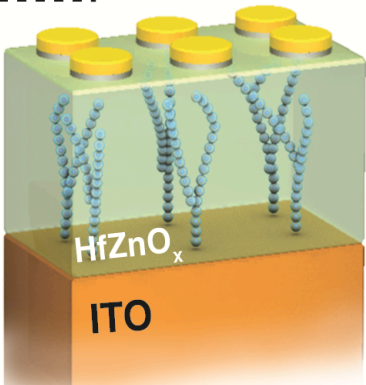

(c)

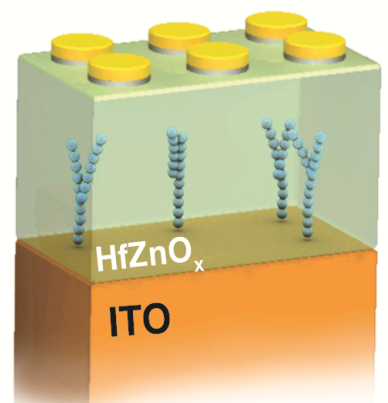

(d) 


\section{Supporting Information}

Controlled Multilevel Conductivity States in $\mathrm{HfZnO}_{\mathbf{x}}$ Memristors for Neuromorphic Memory Applications

Mrinal K. Hota ${ }^{1}$, Mohamed N. Hedhili ${ }^{1}$, Nimer Wehbe ${ }^{1}$, Martyn A. McLachlan ${ }^{2}$, and Husam N. Alshareef ${ }^{1 *}$

${ }^{1}$ Physical Sciences and Engineering Division, King Abdullah University of Science \& Technology

(KAUST), Thuwal 23955-6900, Saudi Arabia

${ }^{2}$ Department of Materials and Centre for Plastic Electronics, Imperial College London, London

SW7 2AZ, United Kingdom

*E-mail: husam.alshareef@kaust.edu.sa

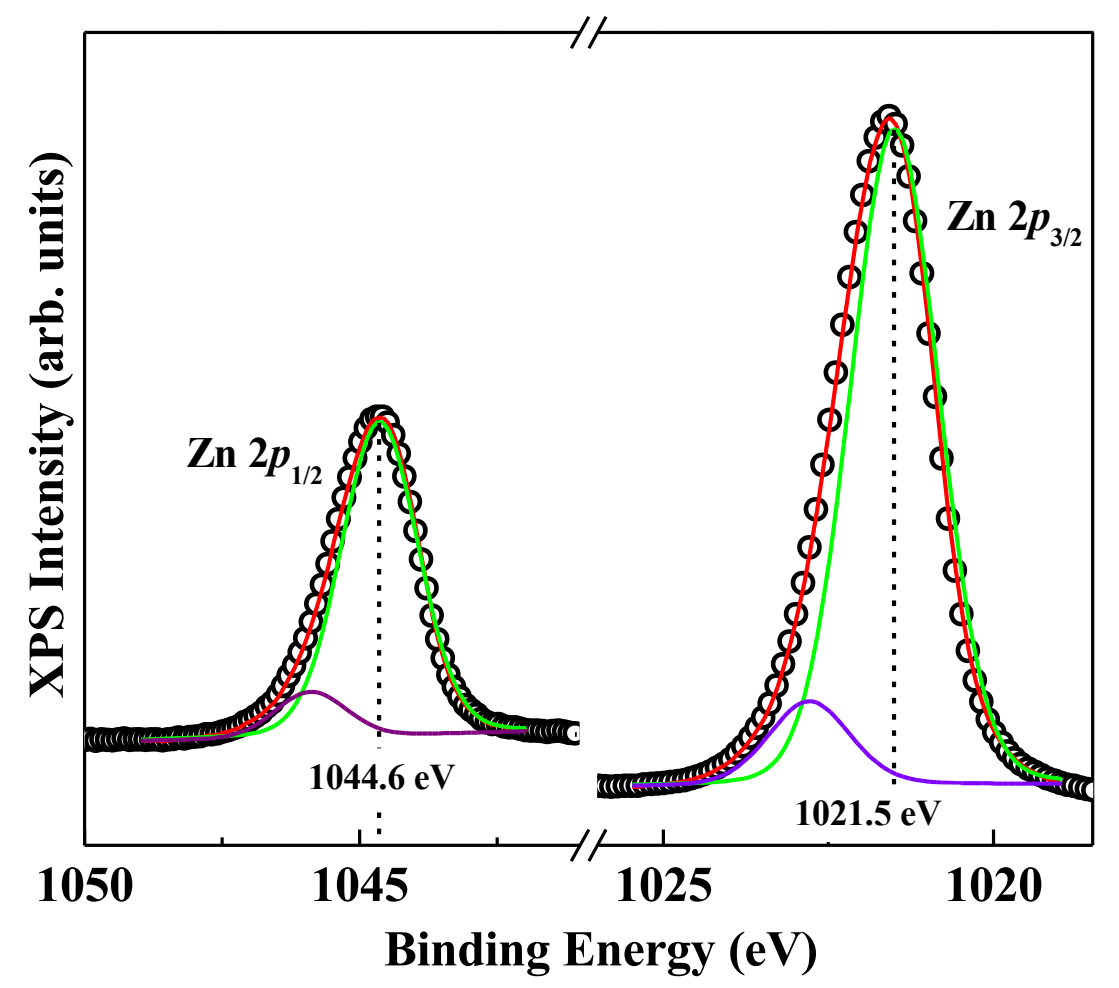

Figure S1: XPS spectra of Zn $2 p$ from ALD deposited pure ZnO sample. 


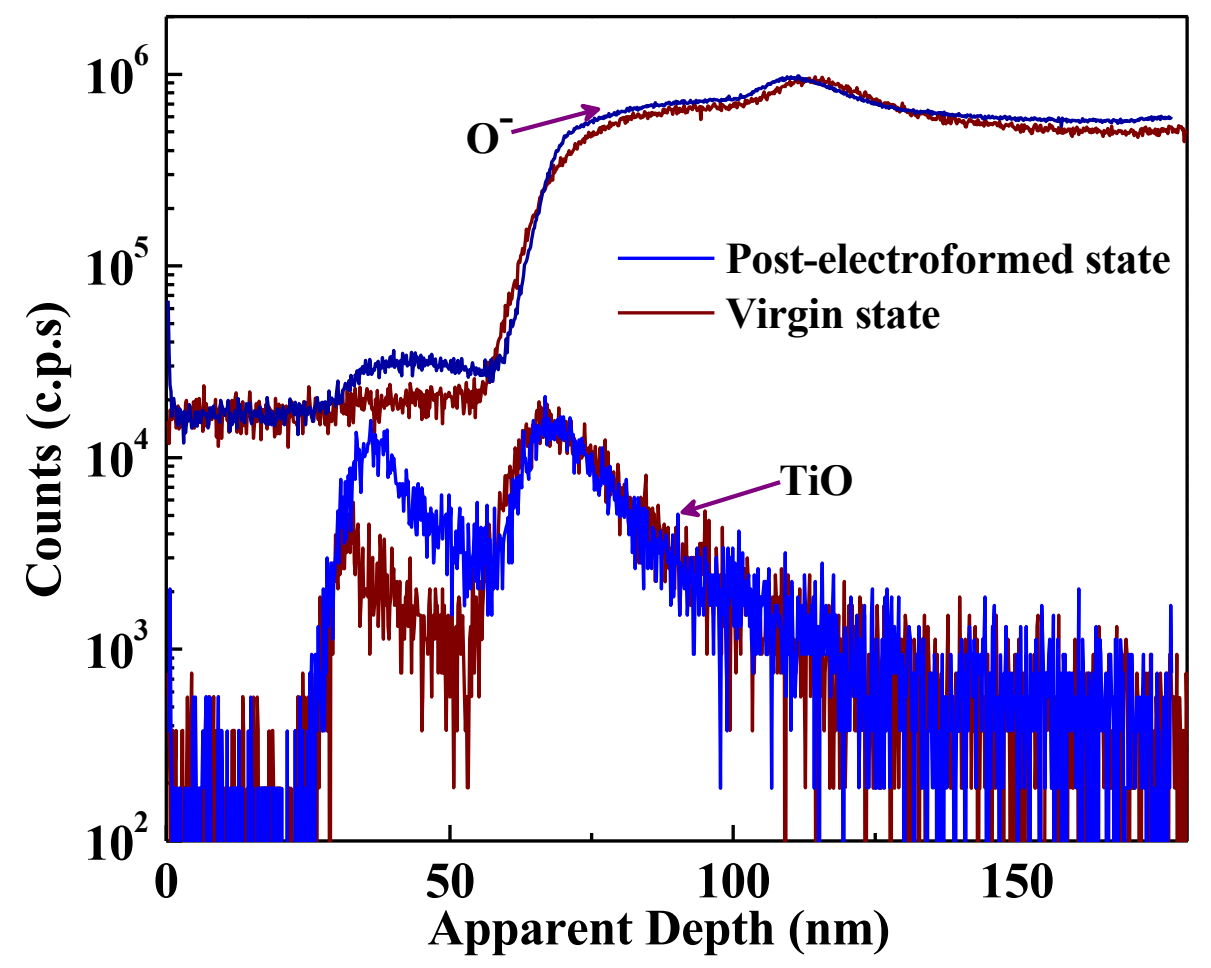

Figure S2: The comparison of $\mathrm{TiO}^{2}$ and $\mathrm{O}^{-}$signals obtained from the SIMS analysis for the sample with virgin and post-electroformed state.

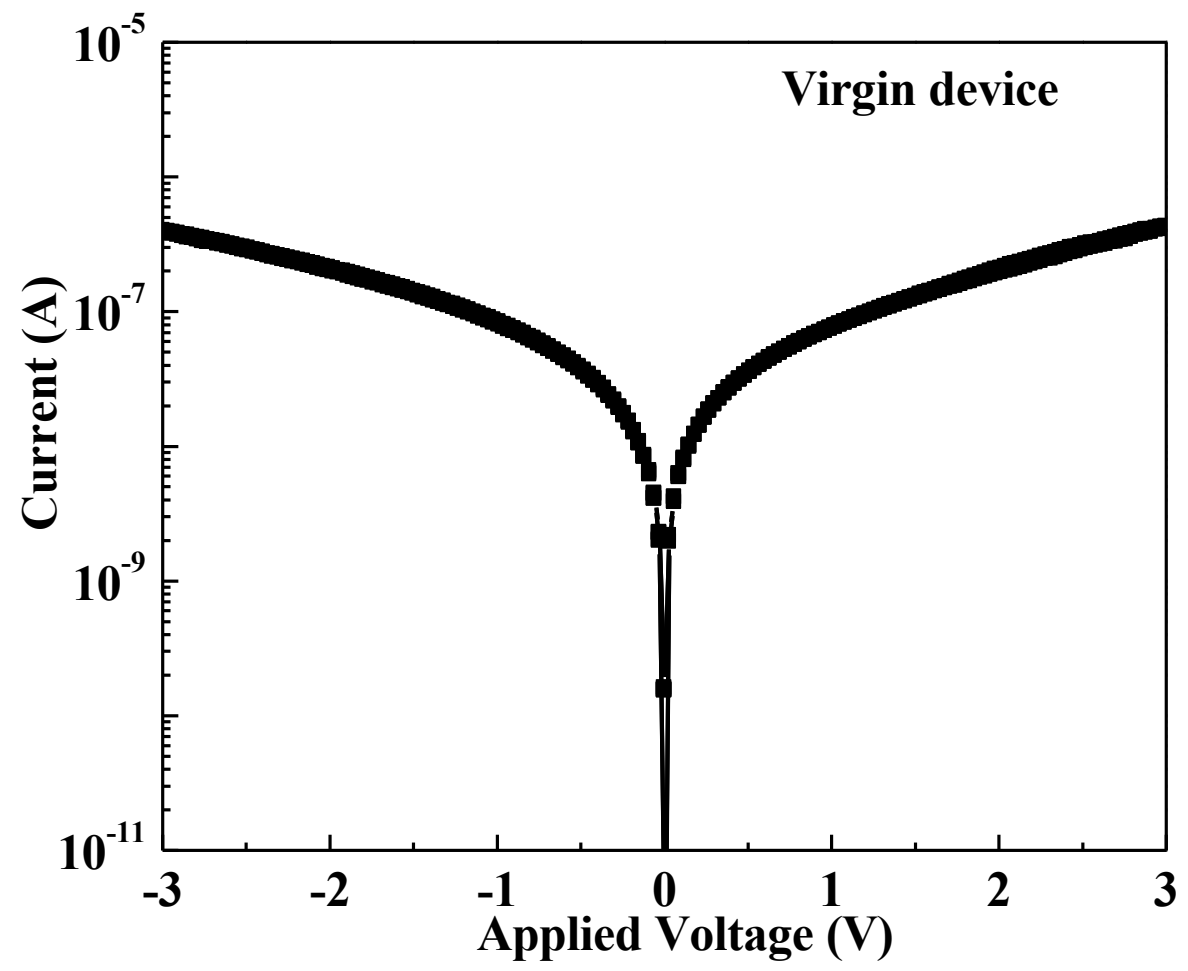

Figure S3: Virgin state current-voltage characteristics obtained from $\mathrm{HfZnO}_{\mathrm{x}}$ based memristor devices. 

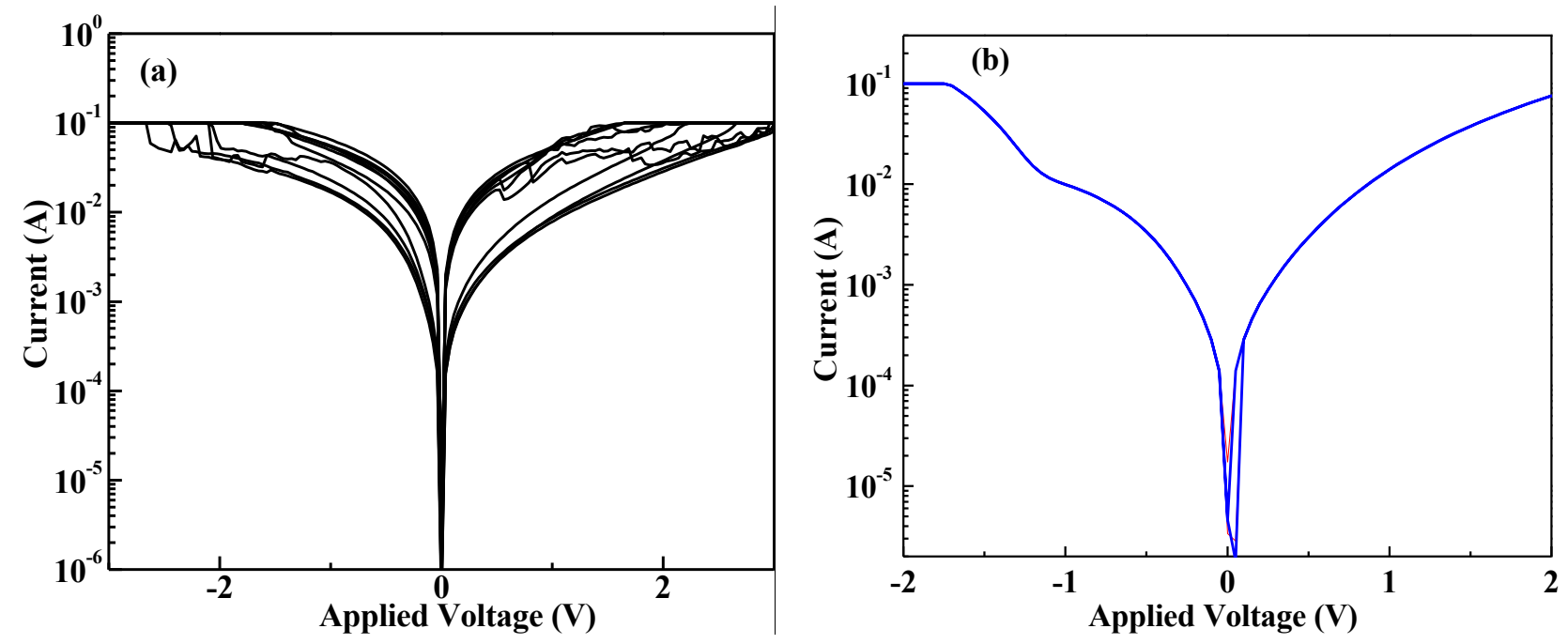

Figure S4: The current-voltage characteristics obtained from (a) ITO/ $\mathrm{HfO}_{2} / \mathrm{Ti} / \mathrm{Au}$ and (b) $\mathrm{ITO} / \mathrm{ZnO} / \mathrm{Ti} / \mathrm{Au}$ memory devices. Poor memory performance was obtained from this device structures.
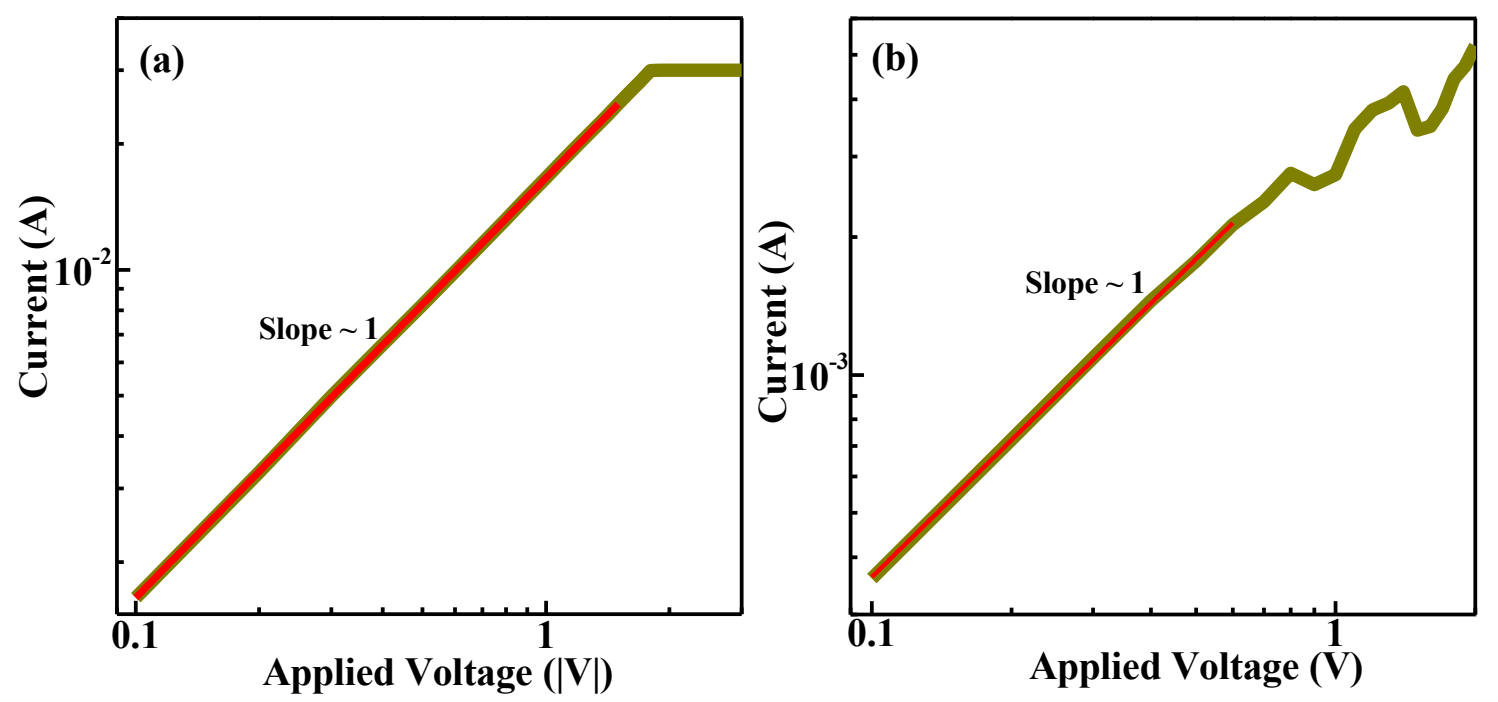

Figure S5: The current conduction process in the (a) negative and (b) positive sides for the LRS state of the memory device. 


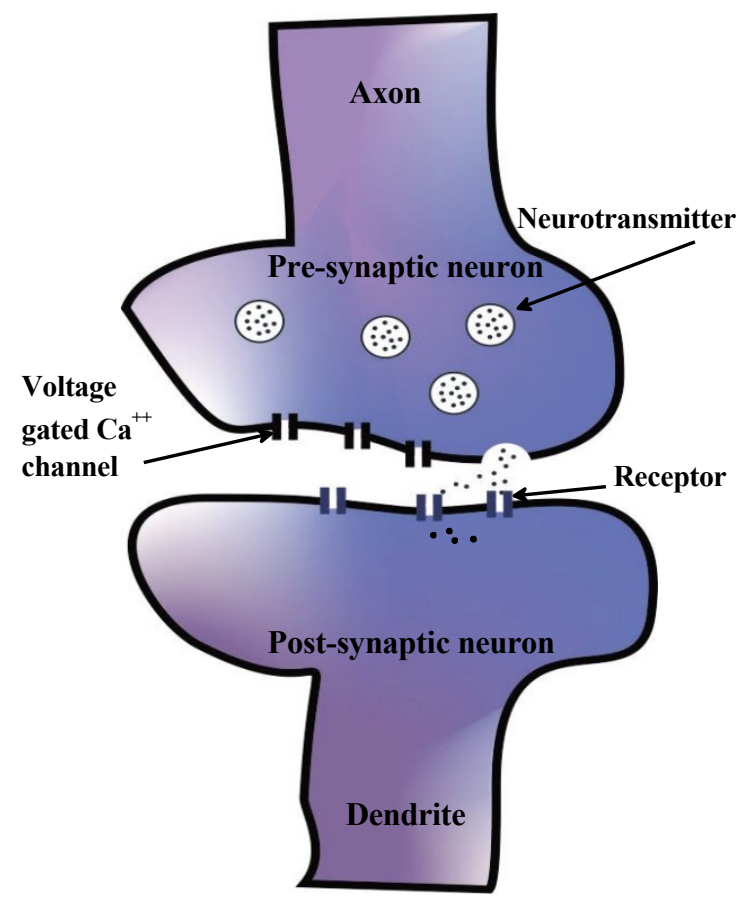

Figure S6: Schematic diagram of the biological synapse. 
